\title{
EFICIÊNCIA DE UTILIZAÇÃO DE NUTRIENTES E SUSTENTABILIDADE DA PRODUÇÃO EM PROCEDÊNCIAS DE Eucalyptus grandis E Eucalyptus saligna EM SÍTIOS FLORESTAIS DO ESTADO DE SÃO PAULO ${ }^{1}$
}

\author{
Reynaldo Campos Santana², Nairam Félix de Barros $^{3,4}$ e Júlio Cézar Lima Neves ${ }^{3}$
}

\begin{abstract}
RESUMO - A eficiência de utilização de nutrientes $(E U N)$ e o crescimento de três procedências de Eucalyptus grandis e de três de E. saligna foram avaliados, aos 78 meses de idade, nos municípios de Angatuba, Itapetininga, Paraibuna, São Miguel Aracanjo e São José dos Campos, Estado de São Paulo. Houve variação na produção de biomassa entre os municípios, sendo as maiores produtividades observadas em São Miguel Aracanjo, seguido por Paraibuna, com os demais municípios constituindo um terceiro grupo. Dentro dos municípios não houve diferença entre materiais genéticos na produção de biomassa, exceto em São Miguel Arcanjo. Os materiais genéticos mostraram variações na $E U N$ entre locais. Observou-se maior $E U N$ de E. grandis em relação ao E. saligna para $\mathrm{N}$, $\mathrm{P}$ e $\mathrm{Mg}$, não havendo diferenças apreciáveis de eficiência entre as duas espécies para Ca e K. A alta $E U N$ não está necessariamente ligada à alta produtividade. $\mathrm{O} \mathrm{Ca}$ e o $\mathrm{K}$ são os nutrientes que mais poderão limitar a produtividade do próximo ciclo, quando se considera a colheita do tronco. Entretanto, esta limitação pode ser substancialmente reduzida, principalmente para o $\mathrm{Ca}$, se a colheita for realizada apenas do lenho.
\end{abstract}

Palavras-chave: Eucalipto, biomassa, nutrição mineral e $E U N$.

\section{NUTRIENT USE EFFICIENCY AND PRODUCTION SUSTAINABILITY OF Eucalyptus grandis AND Eucalyptus saligna PROVENANCES AT DIFFERENT SITES OF SÃO PAULO STATE}

\begin{abstract}
Nutrient use efficiency and growth of three provenances of Eucalyptus grandis and three of E. saligna were evaluated, at 78 months of age, on various sites located in the counties of Angatuba, Itapetininga, Paraibuna, São Miguel Arcanjo and São José dos Campos, in the state of São Paulo, Brazil. There was a variation in biomass production among sites, with the highest yield being obtained in São Miguel Arcanjo, followed by Paraibuna. The other counties constituted a third group. The genetic materials revealed variations in nutrient use efficiency from site to site. E. grandis showed greater $N, P$, and Mg nutrient use efficiency than $\boldsymbol{E}$. saligna. There were no appreciable differences in efficiency between the two species for Ca and K. High nutrient use efficiency is not necessarily related to high productivity. Ca and Kare the nutrients that could most limit productivity of the next rotation, when harvesting the stem. However, this limitation can be substantially reduced, especially for $\mathrm{Ca}$, if only stemwood is harvested.
\end{abstract}

Key words: $\quad$ Eucalypt, biomass, mineral nutrition, nutrient use efficiency.

\section{INTRODUÇÃO}

O Brasil possui 5 milhões de hectares de florestas plantadas, sendo 3 milhões de hectares de eucalipto
(Sociedade...-SBS, 2000), o que coloca o país em lugar de destaque entre os que cultivam esta essência florestal. Os Estados da Bahia, Paraná, Espírito Santo, Minas Gerais, Pará, Rio Grande do Sul e São Paulo são

1 Recebido para publicação em 20.6.2001.

Aceito para publicação em 20.6.2002.

Pesquisa financiada pela CAPES e pela Cia. Suzano de Papel e Celulose.

2 Doutor em Solos e Nutrição de Plantas, DPS/UFV; ${ }^{3}$ Professores do Dep. de Solos da Universidade Federal de Viçosa - UFV, 36571-000 Viçosa-MG; ${ }^{4}$ Bolsista do CNPq. 
responsáveis por $86 \%$ da produção de eucalipto (Instituto...-IBGE, 1998). Essa dispersão geográfica das plantações implica o efeito de diferentes condições fisiográficas, edáficas e climáticas no crescimento do eucalipto (Moreira, 1997). Em decorrência de tal fato, a produtividade das plantações de eucalipto no País varia de 15 a $107 \mathrm{~m}^{3} / \mathrm{ha} /$ ano.

Na década de 90, os programas de seleção de eucalipto no Brasil passaram também a considerar a eficiência de utilização de nutrientes $(E U N)$ como critério para escolha de genótipos superiores, além da produtividade, qualidade da madeira, forma da árvore e resistência a doenças.

A EUN pode variar dentro de uma espécie de planta em função das diferenças genotípicas que podem ocorrer e da interação genótipo-ambiente. Estas diferenças podem alterar a capacidade de absorção, transporte e utilização dos nutrientes pelas plantas (Steenbjerg \& Jakobsen, 1963; Marschner, 1997). Vários estudos abordam a dinâmica de nutrientes e a $E U N$ em plantios de eucalipto, e informações relevantes podem ser encontradas na literatura (Barros et al., 1990; Barros \& Novais, 1996; Gonçalves et al., 1997; Santana et al., 2000). A EUN para um determinado nutriente pode variar à medida que sua disponibilidade no solo seja alterada; em geral a EUN pela planta aumenta com a redução de sua disponibilidade no solo (Barros et al., 1986). Barros et al. (1990) verificaram, também, que a alta eficiência para um dado nutriente não está associada à alta eficiência para os demais nutrientes.

A sustentabilidade de um ecossistema florestal, entre outros fatores, está associada com a estabilidade do balanço de nutrientes a curto, médio e longo prazo. A circulação de nutrientes depende dos ciclos geoquímico, biogeoquímico e bioquímico (Switzer \& Nelson, 1972), ciclos estes que influenciarão e serão influenciados pela $E U N$. O emprego de técnicas de manejo que optem por plantas mais eficientes em utilizar nutrientes, que conservem ao máximo os resíduos das culturas no sítio, que realizem o mínimo possível de intervenções antrópicas e cujo ciclo de crescimento seja longo o suficiente para permitir a máxima eficiência da ciclagem de nutrientes levará à maior conservação do ecossistema de florestas plantadas.

O reconhecimento das diferenças na eficiência de utilização de nutrientes é importante na seleção de técnicas de manejo do solo e de manejo florestal, para manter a capacidade produtiva do sítio ao longo das rotações (Teixeira et al., 1989).

Os objetivos do presente trabalho foram avaliar a $E U N$ e estimar o balanço de nutrientes em povoamentos de diversas procedências de $E$. grandis $\mathrm{W}$. Hill ex Maiden e E. saligna $\mathrm{Sm}$., cultivadas em diferentes sítios florestais no Estado de São Paulo.

\section{MATERIAL E MÉTODOS}

Previamente à instalação dos experimentos, foram selecionados sítios com plantios de eucalipto que apresentavam diferentes produtividades florestais, localizados em cinco municípios do Estado de São Paulo, cujas características edafoclimáticas e adubações aplicadas estão apresentadas nos Quadros 1 e 2, respectivamente. As adubações corresponderam ao que se adotava operacionalmente na empresa, em cada local, à época de instalação dos ensaios.

Utilizou-se o delineamento de blocos casualizados nos cinco locais, tendo sido testados seis materiais genéticos, com quatro repetições, por local. O plantio foi realizado entre dezembro de 1984 e janeiro de 1985. As parcelas foram constituídas por 49 plantas, no espaçamento de 3,0 × 1,8 m, e adotou-se bordadura simples, o que resultou em 25 plantas úteis por repetição.

A avaliação do crescimento das plantas foi realizada aos 6,5 anos após o plantio, medindo-se a altura (ALT) e o diâmetro a 1,3 m de altura do solo (DAP). Em cada local, uma trincheira foi aberta para coleta de solo e sua classificação. As análises químicas e físicas das amostras de solos foram efetuadas, seguindo os métodos descritos por Raij \& Quaggio (1983) e EMBRAPA (1979). Foram também determinados os teores totais de fósforo, potássio, cálcio e magnésio, segundo Page (1982). O cálcio e o magnésio foram determinados por espectrometria de absorção atômica, o potássio por fotometria de chama e o fósforo por colorimetria (Quadro 3).

Os materiais genéticos utilizados consistiram de três procedências de E. grandis (Pine Creek S.F., Monte Lewis e Salto Duratex) e três de E. saligna (Pine Creek S.F., Cascade S.F. e Itatinga). Para cada material genético e local foram estabelecidas cinco classes diamétricas (valor da amplitude dos diâmetros divido por cinco), para coleta do material vegetal. Abateu-se pelo menos uma árvore representativa do diâmetro médio de cada classe, por sítio e material genético, o que resultou em 
265 árvores-amostra. Foi determinado o volume real, com e sem casca, de cada árvore, utilizando a fórmula de Smalian (Campos et al., 1985). As amostras de folhas, galhos, casca e lenho, de cada árvore amostrada, foram homogeneizadas, por material genético, independentemente de repetição, e por local, resultando em uma am ostra composta $¥ 200 \mathrm{~g})$, que foi seca em estufa a $65^{\circ} \mathrm{C}$. Ajustaram-se equações de regressão, relacionando a biomassa de folha, galho, casca e lenho e volume com e sem casca, das árvores amostradas, com a variável independente conjugada LN(DAP ${ }^{2 *}$ ALT) conforme Santana (1999). Em seguida, calculou-se a produção de biomassa dos componentes das árvores amostradas por unidade de área. Estes dados foram submetidos à análise de variância e as médias foram comparadas pelo teste de Tukey, a 5\% de probabilidade.

Quadro 1 - Características edafoclimáticas ${ }^{1 /}$ dos municípios do Estado de São Paulo utilizadas nos experimentos Table 1 - Climatic characteristics $\underline{1}$ / of the counties in the state of São Paulo used in the experiment

\begin{tabular}{|l|c|c|c|c|c|}
\hline \multicolumn{1}{|c|}{ Característica } & \multicolumn{3}{|c|}{ Município } \\
\cline { 2 - 6 } & Itapetininga & $\begin{array}{c}\text { São Miguel } \\
\text { Arcanjo }\end{array}$ & Angatuba & $\begin{array}{c}\text { São José dos } \\
\text { Campos }\end{array}$ & Paraibuna \\
\hline Altitude $(\mathrm{m})$ & 610 & 715 & 649 & 750 & 1.000 \\
Relevo local & plano & plano & plano & ondulado & ondulado \\
Declividade $(\mathrm{cm} / \mathrm{m})^{2 /}$ & $0-5$ & $0-3$ & $0-3$ & $12-25$ & $5-12$ \\
Isotermas médias anuais $\left({ }^{\circ} \mathrm{C}\right)$ & $19-20$ & $18-19$ & $19-20$ & $19-20$ & $17-18$ \\
Isotermas médias janeiro $\left({ }^{\circ} \mathrm{C}\right)$ & $22-23$ & $21-22$ & $22-23$ & $21-22$ & $20-21$ \\
Isotermas médias julho $\left({ }^{\circ} \mathrm{C}\right)$ & $15-16$ & $14-15$ & $15-16$ & $15-16$ & $14-15$ \\
Precipitação pluviométrica média $(\mathrm{mm})$ & $<1200$ & 1400 & 1300 & 1400 & 1500 \\
Deficiência hídrica (mm) & $5-10$ & 0 & $0-5$ & $0-5$ & 0 \\
Classificação climática $(\mathrm{Köppen})$ & $\mathrm{Cfa}$ & $\mathrm{Cfb}$ & $\mathrm{Cfa}$ & $\mathrm{Cfb}$ & $\mathrm{Cfb}$ \\
Latitude & $23^{\circ} 32^{\prime} \mathrm{S}$ & $23^{\circ} 51^{\prime} \mathrm{S}$ & $23^{\circ} 17$ 'S & $23^{\circ} 18^{\prime} \mathrm{S}$ & $23^{\circ} 31^{\prime} \mathrm{S}$ \\
Longitude & $47^{\circ} 51^{\prime} \mathrm{W}$ & $47^{\circ} 51^{\prime} \mathrm{W}$ & $48^{\circ} 28^{\prime} \mathrm{W}$ & $45^{\circ} 53^{\prime} \mathrm{W}$ & $45^{\circ} 39^{\prime} \mathrm{W}$ \\
\hline
\end{tabular}

${ }^{1 /}$ Fonte: Alfonsi \& Camargo (1988).

2/ Declividade da área experimental.

Quadro 2 - Adubações de plantio utilizadas nos experimentos Table 2 - Fertilizer regimes used in the experiments

\begin{tabular}{|c|c|c|c|c|c|}
\hline \multirow{3}{*}{ Fonte } & \multicolumn{5}{|c|}{ Município } \\
\hline & Itapetininga & $\begin{array}{c}\text { São Miguel } \\
\text { Arcanjo }\end{array}$ & Angatuba & $\begin{array}{c}\text { São José dos } \\
\text { Campos }\end{array}$ & Paraibuna \\
\hline & \multicolumn{5}{|c|}{$(\mathrm{kg} / \mathrm{ha})$} \\
\hline Fosfato-de-araxá & 1.000 & 1.000 & - & - & - \\
\hline FAPS & - & - & - & 278 & - \\
\hline Superfosfato triplo & 167 & - & 167 & - & - \\
\hline Termofosfato & - & - & - & - & 370 \\
\hline NPK 06-30-06 & - & - & - & 260 & - \\
\hline NPK $20-00-20$ & 84 & 84 & 84 & 84 & 84 \\
\hline
\end{tabular}

Todos os fertilizantes foram aplicados, de uma única vez, em covas de 40 × 40 x $40 \mathrm{~cm}$, exceto o fosfato-de-araxá, que foi aplicado a lanço em área total, após o preparo do solo ter sido realizado por arado de disco. 
Quadro 3 - Características físicas e químicas dos solos dos sítios estudados Table 3 - Physical and chemical characteristics of soils in the studied sites

\begin{tabular}{|c|c|c|c|c|c|c|c|c|c|c|c|c|c|c|c|c|c|}
\hline \multirow{2}{*}{ Prof. } & \multirow{2}{*}{$\mathrm{pH}$} & \multirow{2}{*}{ MO } & \multicolumn{7}{|c|}{ Trocável } & \multicolumn{4}{|c|}{ Total } & \multirow{2}{*}{ Areia } & \multirow{2}{*}{ Silte } & \multirow{2}{*}{ Argila } & \multirow{2}{*}{$\begin{array}{c}\text { Densidade } \\
\text { do solo }\end{array}$} \\
\hline & & & $\mathrm{P}$ & $\mathrm{K}$ & $\mathrm{Ca}$ & $\mathrm{Mg}$ & $\mathrm{Al}$ & $\mathrm{T}$ & $\mathrm{H}+\mathrm{Al}$ & $\mathrm{P}$ & K & $\mathrm{Ca}$ & $\mathrm{Mg}$ & & & & \\
\hline$(\mathrm{cm})$ & $\mathrm{H}_{2} \mathrm{O}$ & $(\mathrm{dag} / \mathrm{kg})$ & \multicolumn{2}{|c|}{$(\mathrm{mg} / \mathrm{kg})$} & \multicolumn{5}{|c|}{$\left(\mathrm{cmol}_{\mathrm{c}} / \mathrm{kg}\right)$} & \multicolumn{2}{|c|}{$(\mathrm{mg} / \mathrm{kg})$} & \multicolumn{2}{|c|}{$\left(\mathrm{cmol}_{\mathrm{c}} / \mathrm{kg}\right)$} & \multicolumn{3}{|c|}{$(\mathrm{g} / \mathrm{kg})$} & $(\mathrm{g} / \mathrm{cm})$ \\
\hline \multicolumn{18}{|c|}{ São José dos Campos (LVa) } \\
\hline $00-20$ & 4,8 & 2,0 & 3 & 20 & 0,1 & 0,1 & 1,8 & 6,1 & 5,8 & 436 & 556 & 1,1 & 1,8 & 230 & 90 & 680 & 1,18 \\
\hline $20-60$ & 4,7 & 1,9 & 4 & 13 & 0,1 & 0,1 & 1,3 & 5,1 & 4,8 & 391 & 455 & 1,0 & 1,8 & 210 & 90 & 700 & 1,18 \\
\hline $60-120$ & 5,2 & 1,0 & 3 & 8 & 0,1 & 0,1 & 0,4 & 3,8 & 3,6 & 485 & 362 & 0,2 & 1,6 & 180 & 100 & 720 & 1,12 \\
\hline $120-200$ & 4,8 & 0,9 & 3 & 8 & 0,1 & 0,1 & 0,8 & 3,0 & 2,8 & 379 & 440 & 0,3 & 1,3 & 190 & 110 & 700 & 1,13 \\
\hline \multicolumn{18}{|c|}{ Angatuba (LVa) } \\
\hline $00-20$ & 4,5 & 1,0 & 2 & 8 & 0,1 & 0,1 & 0,8 & 2,2 & 2,0 & 262 & 83 & 0,5 & 0,6 & 85 & 3 & 12 & 1,47 \\
\hline $20-60$ & 4,6 & 1,1 & 1 & 5 & 0,1 & 0,1 & 0,8 & 2,1 & 1,9 & 139 & 94 & 0,4 & 0,6 & 85 & 3 & 12 & 1,44 \\
\hline $60-120$ & 4,7 & 0,9 & 1 & 4 & 0,1 & 0,1 & 0,8 & 1,9 & 1,6 & 69 & 342 & 0,6 & 0,9 & 83 & 4 & 14 & 1,36 \\
\hline $120-200$ & 5,3 & 0,9 & 1 & 4 & 0,1 & 0,1 & 0,5 & 1,8 & 1,6 & 131 & 83 & 0,6 & 1,0 & 82 & 3 & 15 & 1,37 \\
\hline \multicolumn{18}{|c|}{ Itapetininga (LVa) } \\
\hline $00-20$ & 4,3 & 1,9 & 7 & 4 & 0,1 & 0,1 & 1,3 & 5,4 & 5,2 & 131 & 83 & 0,4 & 1,3 & 82 & 7 & 11 & 1,32 \\
\hline $20-60$ & 4,4 & 0,9 & 6 & 4 & 0,1 & 0,1 & 0,9 & 3,5 & 3,3 & 99 & 172 & 0,4 & 1,5 & 75 & 7 & 18 & 1,30 \\
\hline $60-120$ & 4,6 & 0,4 & 6 & 4 & 0,1 & 0,1 & 0,6 & 2,4 & 2,2 & 126 & 208 & 0,5 & 1,6 & 71 & 8 & 22 & 1,23 \\
\hline $120-200$ & 4,7 & 0,3 & 5 & 4 & 0,1 & 0,1 & 0,5 & 2,0 & 1,8 & 70 & 208 & 0,5 & 1,6 & 69 & 8 & 23 & 1,30 \\
\hline \multicolumn{18}{|c|}{ Paraibuna (LVa) } \\
\hline $00-20$ & 4,3 & 3,3 & 3 & 22 & 0,1 & 0,1 & 2,5 & 9,7 & 9,5 & 275 & 376 & 0,4 & 1,9 & 45 & 5 & 50 & 1,15 \\
\hline $20-60$ & 4,6 & 1,7 & 3 & 8 & 0,1 & 0,1 & 1,4 & 5,7 & 5,5 & 391 & 336 & 0,3 & 1,6 & 40 & 5 & 55 & 1,17 \\
\hline $60-120$ & 4,6 & 1,5 & 3 & 7 & 0,1 & 0,1 & 1,2 & 5,3 & 5,0 & 391 & 344 & 0,3 & 1,6 & 39 & 5 & 56 & 1,14 \\
\hline $120-200$ & 4,8 & 1,1 & 2 & 4 & 0,1 & 0,1 & 0,4 & 3,1 & 2,9 & 303 & 321 & 0,4 & 1,4 & 34 & 7 & 59 & 1,20 \\
\hline \multicolumn{18}{|c|}{ São Miguel Arcanjo (LE) } \\
\hline $00-20$ & 4,6 & 4,7 & 3 & 23 & 0,2 & 0,3 & 3,1 & 14,1 & 13,5 & 436 & 440 & 1,6 & 7,1 & 4 & 14 & 82 & 0,97 \\
\hline $20-60$ & 4,9 & 3,8 & 3 & 18 & 0,2 & 0,3 & 2,2 & 10,9 & 10,4 & 395 & 499 & 1,9 & 7,3 & 3 & 11 & 86 & 1,00 \\
\hline $60-120$ & 5,4 & 1,6 & 3 & 8 & 0,1 & 0,2 & 0,4 & 4,3 & 4,0 & 353 & 689 & 1,7 & 7,3 & 4 & 7 & 90 & 1,00 \\
\hline $120-200$ & 5,3 & 0,9 & 4 & 8 & 0,1 & 0,1 & 0,1 & 3,0 & 2,8 & 379 & 780 & 0,9 & 6,9 & 5 & 8 & 87 & 0,93 \\
\hline
\end{tabular}

LVa - Latossolo Vermelho-Amarelo e LV - Latossolo Vermelho.

Após analisar o nitrogênio, fósforo, potássio, cálcio e magnésio, de acordo com Sarruge \& Haag (1974), estimaram-se os conteúdos totais destes nutrientes nos componentes das árvores, multiplicando-se as concentrações de nutrientes pelas respectivas quantidades de matéria seca.

Calculou-se a eficiência de utilização dos nutrientes (EUN) para produção de matéria seca do tronco das árvores pela metodologia proposta por Barros et al. (1986), ou seja, pela razão entre a biomassa seca do tronco e o conteúdo de nutrientes acumulados no tronco.

Estimou-se o número potencial de cortes $(N C)$ (Barros et al., 1986) com base no conteúdo de nutrientes acumulados na planta (aos 6,5 anos de idade) e no conteúdo de nutrientes do solo, em formas trocáveis e em formas totais, considerando a camada de 0 a $100 \mathrm{~cm}$ de profundidade, da seguinte forma: 1) $N C=\frac{\text { nutriente no solo }+ \text { nutriente na copa }}{\text { nutriente no tronco }}$

2) $N C=\frac{\text { nutriente no solo }+ \text { nutriente na copa }+ \text { nutriente na casca }}{\text { nutriente } \text { no lenho }}$

\section{RESULTADOS E DISCUSSÃO}

Os materiais genéticos diferiram consideravelmente quanto à eficiência de utilização de nutrientes para produção de biomassa de tronco, variando, também, com o sítio (Quadros 4 e 5). As maiores produtividades não foram acompanhadas de maiores valores de eficiência, 
fato este desejável para a melhor sustentabilidade da produção florestal. A $E U N$ decresceu na seguinte ordem: $\mathrm{P}>\mathrm{Mg}>\mathrm{K}>\mathrm{N}>\mathrm{Ca}$, sendo esta a mesma tendência observada por Santana et al. (2000). A variação na $E U N$ pode ter ocorrido por vários fatores. Entre estes podem ser mencionadas as características intrínsecas do material genético; a não-obtenção do equilíbrio nutricional ótimo ou crítico entre solo, planta e todos os nutrientes, ou seja, pode ter ocorrido limitação de um ou mais nutrientes disponíveis; e as relações hídricas.

Quadro 4 - Biomassa e eficiência de utilização de nutrientes (EUN) de nutrientes no tronco, para $E$. grandis e $E$. saligna, aos 78 meses de idade, cultivados em cinco municípios do Estado de São Paulo

Table 4 - Biomass and nutrient use efficiency of the stem of 78-month-old $\boldsymbol{E}$. grandis and $\boldsymbol{E}$. saligna, in five sites in the state of São Paulo

\begin{tabular}{|c|c|c|c|c|c|c|}
\hline \multirow{3}{*}{ Espécie/Procedência } & \multirow{2}{*}{ Biomassa } & \multicolumn{5}{|c|}{$E U N$} \\
\hline & & $\mathrm{N}$ & $\mathrm{P}$ & $\mathrm{K}$ & $\mathrm{Ca}$ & $\mathrm{Mg}$ \\
\hline & $(\mathrm{t} / \mathrm{ha})$ & \multicolumn{5}{|c|}{$\mathrm{kg}$ de biomassa/kg de nutriente } \\
\hline \multicolumn{7}{|c|}{ São José dos Campos } \\
\hline E. grandis Pine Creek & $1.04,9$ a & 867 & 8.325 & 1.197 & 478 & 5.828 \\
\hline E. grandis Monte Lewis & $1.01,3 \mathrm{a}$ & 788 & 8.965 & 1.024 & 347 & 4.543 \\
\hline E. grandis Salto Duratex & $1.05,0 \quad \mathrm{a}$ & 763 & 8.400 & 1.234 & 376 & 5.385 \\
\hline E. saligna Pine Creek & 90,1 a & 777 & 8.343 & 1.475 & 620 & 3.173 \\
\hline E. saligna Cascade & 87,0 a & 938 & 6.850 & 1.179 & 723 & 3.295 \\
\hline E. saligna Itatinga & 80,2 a & 672 & 7.035 & 1.132 & 550 & 3.730 \\
\hline $\mathrm{CV}(\%)$ & 23,9 & & & & & \\
\hline \multicolumn{7}{|c|}{ Angatuba } \\
\hline E. grandis Pine Creek & 105,9 a & 768 & 5.724 & 412 & 439 & 2.302 \\
\hline E. grandis Monte Lewis & 95,3 a & 817 & 5.707 & 385 & 453 & 2.108 \\
\hline E. grandis Salto Duratex & 121,5 a & 881 & 5.599 & 365 & 447 & 2.355 \\
\hline E. saligna Pine Creek & 88,4 a & 716 & 4.250 & 375 & 368 & 1.619 \\
\hline E. saligna Cascade & 86,5 a & 856 & 4.971 & 412 & 318 & 1.856 \\
\hline E. saligna Itatinga & $95,4 \quad \mathrm{a}$ & 708 & 4.148 & 395 & 405 & 1.889 \\
\hline CV $(\%)$ & 15,6 & & & & & \\
\hline \multicolumn{7}{|c|}{ Itapetininga } \\
\hline E. grandis Pine Creek & $109,1 \quad \mathrm{a}$ & 694 & 6.234 & 1.702 & 341 & 2.195 \\
\hline E. grandis Monte Lewis & 100,3 a & 572 & 5.040 & 2.417 & 294 & 2.209 \\
\hline E. grandis Salto Duratex & 140,4 a & 894 & 5.639 & 2.000 & 244 & 2.825 \\
\hline E. saligna Pine Creek & 98,5 a & 455 & 4.736 & 3.078 & 347 & 1.750 \\
\hline E. saligna Cascade & 107,9 a & 551 & 5.090 & 1.910 & 272 & 1.951 \\
\hline E. saligna Itatinga & 105,5 a & 499 & 4.668 & 1.549 & 315 & 2.010 \\
\hline CV (\%) & 16,7 & & & & & \\
\hline \multicolumn{7}{|c|}{ Paraibuna } \\
\hline E. grandis Pine Creek & 166,4 a & 579 & 7.740 & 335 & 363 & 2.658 \\
\hline E. grandis Monte Lewis & $129,1 \quad \mathrm{a}$ & 631 & 7.777 & 317 & 313 & 2.062 \\
\hline E. grandis Salto Duratex & 134,4 a & 533 & 7.551 & 396 & 478 & 2.683 \\
\hline E. saligna Pine Creek & 143,5 a & 518 & 7.397 & 447 & 367 & 1.729 \\
\hline E. saligna Cascade & $139,0 \quad \mathrm{a}$ & 556 & 7.514 & 437 & 299 & 1.455 \\
\hline E. saligna Itatinga & $138,6 \mathrm{a}$ & 537 & 8.942 & 358 & 327 & 1.860 \\
\hline CV $(\%)$ & 18,4 & & & & & \\
\hline \multicolumn{7}{|c|}{ São Miguel Arcanjo } \\
\hline E. grandis Pine Creek & $183,2 \mathrm{ab}$ & 651 & 7.763 & 1.081 & 263 & 2.678 \\
\hline E. grandis Monte Lewis & $174,3 \quad b$ & 740 & 8.145 & 1.179 & 210 & 2.113 \\
\hline E. grandis Salto Duratex & $206,3 \mathrm{ab}$ & 688 & 9.128 & 909 & 238 & 2.142 \\
\hline E. saligna Pine Creek & $138,6 \mathrm{c}$ & 627 & 8.230 & 1.366 & 202 & 1.241 \\
\hline E. saligna Cascade & 214,3 a & 652 & 7.879 & 1.411 & 252 & 1.990 \\
\hline E. saligna Itatinga & $175,0 \quad \mathrm{~b}$ & 641 & 9.211 & 878 & 221 & 1.856 \\
\hline CV (\%) & 8,3 & & & & & \\
\hline
\end{tabular}

Valores seguidos por uma mesma letra na coluna não diferem estatísticamente entre si, a $5 \%$ de probabilidade, pelo teste de Tukey. 
Em Itapetininga e Angatuba, têm-se solos com menor fator de capacidade de fósforo e menor retenção de umidade em relação aos solos dos demais sítios, em conseqüência do maior teor de areia desses solos em relação aos demais. De modo geral, foi observada eficiência menor para fósforo, independentemente do material genético, portanto ocorrerá relativamente maior exportação de nutrientes (Quadros 4 e 5) por quantidade de madeira produzida nesses sítios.

Em Itapetininga, os valores de $E U N$ de potássio (Quadro 4) foram bem elevados, em comparação com o valor crítico (Quadro 5). Deve-se considerar que o valor crítico para $E U N$ foi determinado em outras regiões em idades mais avançadas do que a considerada neste estudo. A análise do solo (Quadro 3) demonstra que o potássio pode estar limitando a produtividade em Itapetininga, pois seu teor no solo encontrava-se muito abaixo do nível crítico de manutenção (Novais et al., 1986). Essa hipótese é suportada também pelos nítidos sintomas visuais de deficiências de potássio nas folhas, observados nas brotações de todos os materiais genéticos. Em Angatuba observaram-se os mesmos sintomas de deficiências nas brotações, e em função da similaridade entre este sítio e o de Itapetininga esperava-se que resultados semelhantes para $E U N$ e $\mathrm{K}$ ocorressem, no entanto o resultado foi oposto. Neste sítio, os teores de K nas formas disponível e total são os mais baixos dentre os solos estudados, exceto na camada de $75-120 \mathrm{~cm}$, onde o teor total é mais elevado. Os baixos valores de $E U N$ não são compatíveis com os sintomas visuais observados. Nessa região foram acumulados, em média, cerca de $224 \mathrm{~kg} / \mathrm{ha}$ de $\mathrm{K}$ no tronco das árvores, enquanto em São José dos Campos e Itapetininga, com produções similares à de Angatuba, a quantidade de $\mathrm{K}$ acumulada foi de 98 e $112 \mathrm{~kg} / \mathrm{ha}$, respectivamente. Ressalta-se que em São José dos Campos tanto os teores trocáveis como os totais foram mais elevados do que em Angatuba, o que demonstra a importância e a necessidade da identificação mineralógica dos componentes da fração argila do solo para o melhor entendimento dos fatos aqui observados. Uma hipótese é que durante o ciclo da cultura havia maior disponibilidade de potássio no sítio de Angatuba, que teria sido reduzida ao longo dos anos em razão da sua absorção pelas plantas.

O maior valor de $E U N$ de potássio, avaliado ao final de um ciclo de corte em Itapetininga (Quadro 4), permite inferir sobre a provável redução da produtividade no ciclo seguinte, pois uma $E U N$ extremamente alta ao final de um ciclo em relação à $E U N$ crítica indica que o nutriente

R. Árvore, Viçosa-MG, v.26, n. 4, p.447-457, 2002 avaliado pode ter limitado o crescimento na rotação corrente e limitará o crescimento da rotação ou ciclo subseqüente, a menos que o nutriente seja suprido via fertilizante; uma $E U N$ satisfatória de um determinado nutriente ao final de um ciclo não necessariamente indica que este nutriente não limitará a segunda rotação ou do próximo ciclo, pois para o adequado crescimento da nova rotação é necessário que o solo tenha o nutriente, ou que ele seja adicionado via fertilização, em quantidades suficientes para atingir a produtividade desejada, e que este nutriente esteja disponível para a planta.

De modo geral, observou-se que as procedências de E. grandis apresentaram valores de eficiência nutricional um pouco mais elevados para fósforo e magnésio do que as de E. saligna (Quadro 5), não havendo diferenças apreciáveis de eficiência, entre os materiais genéticos, para os demais nutrientes analisados, independentemente do método utilizado para o cálculo da eficiência.

Em São José dos Campos, os valores absolutos das EUN de magnésio, principalmente para o E. grandis (Quadro 4), comparados ao valor crítico de EUN (Quadro 5), foram bem elevados, o que indica que nesse sítio as procedências de E. grandis foram muito eficientes na utilização do nutriente. Tal fato não foi observado em qualquer dos outros sítios. Os teores trocáveis e totais do elemento no solo (Quadro 3), comparados aos acumulados na planta, levaram a valores elevados. Assim, os valores elevados de EUN podem estar mais relacionados à falta de água para transporte do magnésio ou ao eventual reduzido volume de solo explorado pelas raízes do que à sua falta no solo.

A estimativa do número de cortes para os vários materiais genéticos, em cada local e nutriente, foi semelhante, mas detectam-se grandes variações entre locais e nutrientes (Quadro 6). Por isto, serão considerados apenas os valores médios na discussão sobre este assunto.

A EUN para um determinado nutriente pode variar à medida que sua disponibilidade no solo varia; em geral, a EUN pela planta aumenta com a redução de sua disponibilidade no solo (Barros et al., 1986). Para estimar a produtividade do próximo ciclo, foi assumido que não ocorrerá alteração na $E U N$ em relação ao ciclo anterior e que a entrada se igualará à saída no sistema. Portanto, de modo geral, o potássio e o cálcio são os principais nutrientes, na forma disponível, que poderão limitar a produtividade no próximo ciclo (Quadro 7), pois o valor 
Quadro 5 - Valores médios de biomassa, eficiência de utilização de nutrientes ( $E U N)$, $E U N$ crítico, média e amplitude dos valores de $E U N$ no tronco de $E$. grandis e $E$. saligna, aos 78 meses de idade, cultivados em cinco municípios do Estado de São Paulo

Table 5 -Average values of biomass, nutrient use efficiency (NUE), NUE critical values and range for stem of 78-month-old E. grandis and E. saligna, in five sites in the state of São Paulo

\begin{tabular}{|c|c|c|c|c|c|c|}
\hline & \multirow{3}{*}{$\begin{array}{c}\text { Biomassa } \\
\text { (t/ha) }\end{array}$} & \multicolumn{5}{|c|}{$E U N$} \\
\hline & & $\mathrm{N}$ & $\mathrm{P}$ & $\mathrm{K}$ & $\mathrm{Ca}$ & $\mathrm{Mg}$ \\
\hline & & \multicolumn{5}{|c|}{ (kg de biomassa/kg de nutriente) } \\
\hline E. grandis Pine Creek & 133,9 & 712 & 7.157 & 945 & 377 & 3.132 \\
\hline E. grandis Monte Lewis & 120,1 & 710 & 7.127 & 1.064 & 323 & 2.607 \\
\hline E. grandis Salto & 141,5 & 752 & 7.263 & 981 & 357 & 3.078 \\
\hline Maior Valor & 206,3 & 894 & 9.128 & 2414 & 478 & 5.828 \\
\hline Menor Valor & 95,3 & 533 & 5.040 & 317 & 210 & 2.062 \\
\hline Valor Médio & 131,8 & 724 & 7.182 & 997 & 352 & 2.939 \\
\hline E. saligna Pine Creek & 111,8 & 619 & 6.595 & 1348 & 381 & 1.902 \\
\hline E. saligna Cascade & 126,9 & 710 & 6.461 & 1070 & 373 & 2.110 \\
\hline E. saligna Itatinga & 118,9 & 611 & 6.801 & 862 & 364 & 2.269 \\
\hline Maior Valor & 214,3 & 938 & 9.211 & 3078 & 723 & 3.730 \\
\hline Menor Valor & 80,2 & 455 & 4.148 & 358 & 202 & 1.241 \\
\hline Valor Médio & 119,2 & 647 & 6.619 & 1093 & 372 & 2.094 \\
\hline EUN Crítico ${ }^{\underline{1} /}$ (Nutricalc) & - & - & 12.000 & 1.000 & 600 & 3.000 \\
\hline
\end{tabular}

1/ Valores adotados como críticos no software NUTRICALC (Barros et al., 1995).

da estimativa do número de cortes foi próximo à unidade, para todos os locais.

Esse quadro pode ser modificado, caso a porção colhida se restrinja ao lenho. Nesta alternativa as relações nutricionais são substancialmente alteradas, aumentando a expectativa da produção, em média, 5, 3, 2, e 1,7 vezes, respectivamente, para cálcio, magnésio, fósforo e potássio, independentemente das espécies. Tal fato seria muito importante para manutenção dos teores de cálcio no solo, pois a maioria dos solos cultivados com eucalipto apresenta baixos teores deste nutriente e, em média, 58\% do total de cálcio absorvido é exportado pela colheita da casca (Quadro 8). Com este cenário, o potássio torna-se o principal nutriente que poderá limitar a produtividade do próximo ciclo.

De modo geral, todos os sítios possuem alto potencial de resposta à fertilização com fósforo, potássio, cálcio e magnésio. Atenção especial deve ser dada ao cálcio, pois a estimativa do número de cortes, considerando os conteúdos totais de cálcio, é muito baixa, atingindo os menores valores em Paraibuna, condição que pode ser razoavelmente melhorada quando a colheita se restringe à madeira do tronco.

Como constatado por outros autores (Teixeira et al., 1989; Morais et al., 1990; Molica, 1992), dificilmente será possível selecionar um material genético que possua alta eficiência na utilização de todos os nutrientes. Assim, o manejador florestal deverá utilizar materiais genéticos que possuam eficiência compatível com a fertilidade dos solos, ou seja, materiais pouco eficientes na absorção de cálcio deveriam ser plantados em solos ricos neste nutriente, ou recebê-lo na forma de adubo ou calcário. Entretanto, se materiais altamente eficientes na absorção de um nutriente forem plantados em solos pobres que não receberem adubação, deverá ocorrer depauperação mais rápida de solo. 
Quadro 6 - Número de cortes previstos, em função da quantidade de nutrientes no solo, para procedências de $E$. grandis e E. saligna, aos 78 meses de idade, cultivados em cinco municípios do Estado de São Paulo

Table 6 -Number of harvests predicted, as a function of the amount of nutrients in the soil, for provenances of 78-month-old E. grandis and E. saligna, in five sites of the state of São Paulo

\begin{tabular}{|c|c|c|c|c|c|c|c|c|}
\hline \multirow{3}{*}{ Procedência } & \multicolumn{4}{|c|}{ Nutriente Disponível } & \multicolumn{4}{|c|}{ Nutriente Total } \\
\hline & $\mathrm{P}$ & $\mathrm{K}$ & $\mathrm{Ca}$ & $\mathrm{Mg}$ & $\mathrm{P}$ & $\mathrm{K}$ & $\mathrm{Ca}$ & $\mathrm{Mg}$ \\
\hline & \multicolumn{8}{|c|}{$\mathrm{kg} / \mathrm{ha}$ (Nutriente no solo* + Copa /Nutriente no tronco) } \\
\hline \multicolumn{9}{|c|}{ São José dos Campos } \\
\hline E. grandis Pine Creek & 3,5 & 2,0 & 1,2 & 8,4 & 410,1 & 57,3 & 7,7 & 136,5 \\
\hline E. grandis Monte Lewis & 4,2 & 1,8 & 1,1 & 7,2 & 456,9 & 50,8 & 6,0 & 110,6 \\
\hline E. grandis Salto Duratex & 3,6 & 2,0 & 1,0 & 7,7 & 414,4 & 58,9 & 6,0 & 125,8 \\
\hline E. saligna Pine Creek & 4,1 & 2,8 & 1,9 & 5,3 & 480,7 & 81,9 & 11,6 & 86,4 \\
\hline E. saligna Cascade & 3,7 & 2,3 & 2,4 & 5,8 & 407,5 & 67,9 & 14,1 & 93,2 \\
\hline E. saligna Itatinga & 4,0 & 2,5 & 2,0 & 7,1 & 455,2 & 70,8 & 11,6 & 114,5 \\
\hline \multicolumn{9}{|c|}{ Angatuba } \\
\hline E. grandis Pine Creek & 1,6 & 0,6 & 1,5 & 4,2 & 103,8 & 9,8 & 5,9 & 27,5 \\
\hline E. grandis Monte Lewis & 1,9 & 0,7 & 1,6 & 4,2 & 115,4 & 10,2 & 6,8 & 28,0 \\
\hline E. grandis Salto Duratex & 1,4 & 0,5 & 1,3 & 3,7 & 88,5 & 7,6 & 5,3 & 24,6 \\
\hline E. saligna Pine Creek & 1,4 & 0,6 & 1,4 & 3,6 & 92,4 & 10,6 & 5,9 & 23,3 \\
\hline E. saligna Cascade & 1,6 & 0,6 & 1,3 & 4,1 & 110,1 & 11,8 & 5,2 & 27,1 \\
\hline E. saligna Itatinga & 1,2 & 0,6 & 1,4 & 3,7 & 83,4 & 10,3 & 6,0 & 25,0 \\
\hline \multicolumn{9}{|c|}{ Itapetininga } \\
\hline E. grandis Pine Creek & 5,1 & 1,0 & 1,1 & 3,6 & 86,6 & 34,1 & 4,0 & 47,6 \\
\hline E. grandis Monte Lewis & 4,5 & 1,6 & 0,9 & 3,8 & 76,4 & 52,8 & 3,7 & 51,9 \\
\hline E. grandis Salto Duratex & 3,6 & 1,0 & 0,6 & 3,5 & 61,1 & 31,2 & 2,2 & 47,4 \\
\hline E. saligna Pine Creek & 4,3 & 1,9 & 1,2 & 3,1 & 72,9 & 68,3 & 4,5 & 41,9 \\
\hline E. saligna Cascade & 4,4 & 1,3 & 0,8 & 3,1 & 71,9 & 38,9 & 3,2 & 42,6 \\
\hline E. saligna Itatinga & 4,0 & 0,9 & 0,9 & 3,3 & 67,2 & 32,1 & 3,7 & 44,8 \\
\hline \multicolumn{9}{|c|}{ Paraibuna } \\
\hline E. grandis Pine Creek & 2,0 & 0,5 & 0,7 & 2,7 & 209,6 & 8,7 & 1,9 & 40,1 \\
\hline E. grandis Monte Lewis & 2,7 & 0,6 & 0,7 & 2,7 & 272,1 & 10,6 & 2,1 & 40,0 \\
\hline E. grandis Salto Duratex & 2,6 & 0,6 & 1,1 & 3,4 & 253,8 & 12,7 & 3,0 & 50,2 \\
\hline E. saligna Pine Creek & 2,2 & 0,7 & 0,8 & 2,0 & 232,1 & 13,4 & 2,2 & 30,2 \\
\hline E. saligna Cascade & 2,3 & 0,7 & 0,7 & 1,7 & 243,6 & 13,6 & 1,9 & 26,3 \\
\hline E. saligna Itatinga & 2,8 & 0,6 & 0,7 & 2,2 & 291,7 & 11,1 & 2,0 & 33,6 \\
\hline \multicolumn{9}{|c|}{ São Miguel Arcanjo } \\
\hline E. grandis Pine Creek & 1,4 & 1,1 & 0,6 & 5,3 & 135,8 & 27,3 & 4,8 & 109,6 \\
\hline E. grandis Monte Lewis & 1,5 & 1,2 & 0,4 & 4,3 & 149,8 & 31,1 & 4,0 & 90,7 \\
\hline E. grandis Salto Duratex & 1,6 & 0,8 & 0,4 & 3,8 & 142,0 & 20,4 & 3,8 & 77,9 \\
\hline E. saligna Pine Creek & 2,0 & 1,7 & 0,5 & 3,3 & 190,4 & 45,4 & 4,8 & 67,1 \\
\hline E. saligna Cascade & 1,4 & 1,3 & 0,5 & 3,5 & 118,3 & 30,5 & 3,9 & 69,7 \\
\hline E. saligna Itatinga & 2,1 & 1,0 & 0,5 & 4,0 & 168,7 & 23,2 & 4,2 & 79,6 \\
\hline
\end{tabular}

* Conteúdo de nutrientes no solo, considerando a camada de 0 a $100 \mathrm{~cm}$ de profundidade. 
Quadro 7 - Número de cortes médio para $E$. grandis e $E$. saligna, aos 78 meses de idade, cultivados em cinco municípios do Estado de São Paulo

Table 7 - Average of number of harvestings of 78-month-old $\boldsymbol{E}$. grandis and $\boldsymbol{E}$. saligna, in five sites in the state of São Paulo

\begin{tabular}{|c|c|c|c|c|c|c|c|c|}
\hline \multirow{3}{*}{ Município } & \multicolumn{4}{|c|}{ Nutriente Disponível } & \multicolumn{4}{|c|}{ Nutriente Total } \\
\hline & $\mathrm{P}$ & $\mathrm{K}$ & $\mathrm{Ca}$ & $\mathrm{Mg}$ & $\mathrm{P}$ & $\mathrm{K}$ & $\mathrm{Ca}$ & $\mathrm{Mg}$ \\
\hline & \multicolumn{8}{|c|}{$\mathrm{kg} / \mathrm{ha}$ (Nutriente no solo* + Copa /Nutriente no tronco) } \\
\hline \multicolumn{9}{|c|}{ E. grandis } \\
\hline São José dos Campos & 3,7 & 2,0 & 1,1 & 7,8 & 427,1 & 55,7 & 6,6 & 124,3 \\
\hline Angatuba & 1,6 & 0,6 & 1,5 & 4,1 & 102,6 & 9,2 & 6,0 & 26,7 \\
\hline Itapetininga & 4,4 & 1,2 & 0,9 & 3,6 & 74,7 & 39,4 & 3,3 & 49,0 \\
\hline Paraibuna & 2,4 & 0,6 & 0,8 & 2,9 & 245,2 & 10,7 & 2,3 & 43,4 \\
\hline São Miguel Arcanjo & 1,5 & 1,0 & 0,5 & 4,5 & 142,5 & 26,3 & 4,2 & 92,7 \\
\hline \multicolumn{9}{|c|}{ E. saligna } \\
\hline São José dos Campos & 3,9 & 2,5 & 2,1 & 6,1 & 447,8 & 73,6 & 12,4 & 98,0 \\
\hline Angatuba & 1,4 & 0,6 & 1,4 & 3,8 & 95,3 & 10,9 & 5,7 & 25,1 \\
\hline Itapetininga & 4,2 & 1,4 & 1,0 & 3,2 & 70,7 & 46,4 & 3,8 & 43,1 \\
\hline Paraibuna & 2,4 & 0,7 & 0,7 & 2,0 & 255,8 & 12,7 & 2,0 & 30,0 \\
\hline São Miguel Arcanjo & 1,8 & 1,3 & 0,5 & 3,6 & 159,1 & 33,0 & 4,3 & 72,2 \\
\hline
\end{tabular}

* Conteúdo de nutrientes no solo, considerando a camada de 0 a $100 \mathrm{~cm}$ de profundidade.

Quadro 8 - Número médio de cortes para E. grandis e E. saligna, aos 78 meses de idade, cultivados em cinco municípios do Estado de São Paulo, considerando a colheita somente do lenho do tronco

Table 8 -Average of number of harvests of 78-month-old $\boldsymbol{E}$. grandis and $\boldsymbol{E}$. saligna, in five sites in the state of São Paulo, taking into account only stemwood harvesting

\begin{tabular}{|c|c|c|c|c|c|c|c|c|}
\hline \multirow{3}{*}{ Município } & \multicolumn{4}{|c|}{ Nutriente Disponível } & \multicolumn{4}{|c|}{ Nutriente Total } \\
\hline & $\mathrm{P}$ & $\mathrm{K}$ & $\mathrm{Ca}$ & $\mathrm{Mg}$ & $\mathrm{P}$ & $\mathrm{K}$ & $\mathrm{Ca}$ & $\mathrm{Mg}$ \\
\hline & \multicolumn{8}{|c|}{$\mathrm{kg} / \mathrm{ha}$ (Nutriente no solo* + Copa + Casca/Nutriente no lenho) } \\
\hline \multicolumn{9}{|c|}{ E. grandis } \\
\hline São José dos Campos & 5,2 & 3,6 & 2,2 & 17,8 & 559,6 & 86,3 & 10,3 & 266,6 \\
\hline Angatuba & 4,2 & 1,2 & 6,5 & 11,7 & 204,8 & 13,2 & 20,3 & 68,7 \\
\hline Itapetininga & 10,1 & 1,5 & 3,6 & 10,3 & 154,0 & 46,0 & 9,6 & 120,2 \\
\hline Paraibuna & 4,1 & 1,4 & 6,1 & 8,1 & 362,2 & 17,0 & 11,9 & 101,9 \\
\hline São Miguel Arcanjo & 2,3 & 1,7 & 3,5 & 12,2 & 191,5 & 35,3 & 14,7 & 224,7 \\
\hline \multicolumn{9}{|c|}{ E. saligna } \\
\hline São José dos Campos & 6,4 & 3,9 & 6,1 & 22,1 & 672,3 & 103,7 & 30,0 & 320,9 \\
\hline Angatuba & 5,1 & 1,2 & 7,0 & 14,2 & 243,4 & 15,4 & 21,8 & 81,9 \\
\hline Itapetininga & 11,4 & 1,9 & 8,5 & 11,5 & 167,8 & 56,8 & 21,9 & 131,0 \\
\hline Paraibuna & 3,8 & 1,4 & 10,1 & 9,1 & 363,7 & 19,1 & 18,6 & 103,3 \\
\hline São Miguel Arcanjo & 2,7 & 1,8 & 4,0 & 10,7 & 208,7 & 40,0 & 16,9 & 185,4 \\
\hline
\end{tabular}

* Conteúdo de nutrientes no solo, considerando a camada de 0 a $100 \mathrm{~cm}$ de profundidade. 


\section{CONCLUSÕES}

1. Os materiais genéticos mostraram variações na $E U N$ entre locais.

2. A alta $E U N$ não está necessariamente ligada à alta produtividade.

3. As procedências de $E$. grandis apresentaram maiores valores de $E U N$ para $\mathrm{P}$ e $\mathrm{Mg}$ que as de E. saligna, não havendo diferenças apreciáveis de $E U N$ para os demais nutrientes.

4. A maior exportação de $P$ foi observada nos sítios de solos com menor fator capacidade deste elemento, independentemente do material genético.

5. O número de cortes estimados para os diversos materiais genéticos, para cada local e nutriente, foi semelhante, embora tenham sido detectadas grandes variações entre locais e nutrientes.

6. O K e o Ca são os nutrientes que poderão limitar a produtividade do próximo ciclo. Essa limitação pode ser substancialmente reduzida, independentemente das espécies, se a colheita se limitar ao lenho.

\section{AGRADECIMENTOS}

À CAPES (Coordenação de Aperfeiçoamento de Pessoal de Ensino Superior), pela bolsa de estudos, e à Cia Suzano de Papel e Celulose, pelo apoio técnico e financeiro.

\section{REFERÊNCIAS BIBLIOGRÁFICAS}

ALFONSI, R. R.; CAMARGO, M. B. P. Característica climática das áreas de propriedade da Cia. Suzano de Papel e Celulose no Estado de São Paulo. São Paulo: Cia. Suzano Papel e Celulose, 1988. (Relatório Interno)

BARROS, N. F.; NOVAIS, R. F. Relação solo-eucalipto. Viçosa, MG: Folha de Viçosa, 1990. 330 p.

BARROS, N. F. et al. Classificação nutricional de sítios florestais - Descrição de uma metodologia. Revista Árvore, v. 10, n. 2, p. 112-120, 1986.

BARROS, N. F.; NOVAIS, R. F.; NEVES, J. C. L. Fertilização e correção do solo para o plantio de eucalipto. In: BARROS, N. F.; NOVAIS, R. F. (Eds.) Relação soloeucalipto. Viçosa, MG: Folha de Viçosa, 1990. p. 127-186.

BARROS, N. F. et al. Nutricalc 2.0 - Sistema para cálculo del balance nutricional y recomendación de fertilizantes para el cultivo de eucalipto. Bosque, v. 16, n. 1, p. 129-131, 1995.
BARROS, N. F.; NOVAIS, R. F. Eucalypt nutrition and fertilizer regimes in Brazil. In: ATTIWILL, P. M.; ADAMS, M. A. (Eds.). Nutrition of eucalyptus. Collingwood: VIC, CISRO, 1996. p. 335-356.

CAMPOS, J. C. C.; TORQUATO, M. C.; RIBEIRO, G. A. Equações para calcular índices de local e incremento da altura de plantações puras de Eucalyptus grandis. Revista Árvore, v. 9, n. 1, p. 1-9, 1985.

\section{EMPRESA BRASILEIRA DE PESQUISA} AGROPECUÁRIA - EMBRAPA. Manual de métodos e análise de solos. Centro Nacional de Pesquisa de Solos. 1.ed. Rio de Janeiro: 1979. 212 p.

GONÇALVES, J. L. M. et al. Soil and stand management for short-rotation plantations. In: NAMBIAR, E. D. S.; BROWN, A. G. (Eds.) Management of soil nutrients and water in tropical plantations forest. Canberra: ACIAR, 1997. p. 379-418. (Monograph, 43).

INSTITUTO BRASILEIRO DE GEOGRAFIA E ESTATÍSTICA - IBGE. Anuário estatístico do Brasil. Brasília: 1998. v. 58.

MOLICA, S. G. Produção de biomassa e eficiência nutricional de híbridos interespecíficos de eucalipto, em duas regiões bioclimáticas de Minas Gerais. Viçosa, MG: Universidade Federal de Viçosa, 1992. 84 p. Tese (Doutorado em Fitotecnia) - Universidade Federal de Viçosa, 1992.

MORAIS, E. J. et al. Biomassa e eficiência nutricional de espécies de eucalipto em duas regiões bioclimáticas de minas gerais. Revista Brasileira de Ciência do Solo, v. 14, p. 353-362, 1990.

MOREIRA, I. P. S. Classificação ecológica do território brasileiro situado entre 16 e $24^{\circ}$ latitude sul e 48 e $39^{\circ} 51^{\prime}$ longitude oeste. Viçosa, MG: Universidade Federal de Viçosa, 1997. 157 p. Tese (Doutorado em Ciência Florestal) - Universidade Federal de Viçosa, 1997.

MARSCHNER, H. Mineral nutrition of higher plants. 2.ed. San Diego: Academic Press, 1997. 889 p.

NOVAIS, R. F.; BARROS, N. F.; NEVES, J. C. L. Interpretação de análise química do solo para o crescimento de Eucalyptus spp. - níveis críticos de implantação e de manutenção. Revista Árvore, v. 10, n. 1, p. 105-111, 1986.

PAGE, L.A. Methods of soil analysis, Part 2: Chemical and microbiological properties. 2. ed. Madison: American Society of Agronomy, 1982. $1159 \mathrm{p}$. 
STEENBJERG, F.; JAKOBSEN, S. T. Plant nutrition and yield curves. Soil Science, v. 95, p. 69-88, 1963.

SANTANA, R. C.; BARROS, N. F.; COMERFORD, N. B. Aboveground biomass, nutrient content, and nutrient use efficiency of eucalypt plantations growing in different sites in Brazil. New Zealand Journal of Forest Science, v. 30, n. $1 / 2$, p. $225-236,2000$.

SANTANA, R. C.; BARROS, N. F.; NEVES, J. C. L. Produção de biomassa e conteúdo de nutrientes de procedências de Eucalyptus grandis e Eucalyptus saligna em alguns sítios florestais do Estado de São Paulo. Scientia Forestalis, n. 56, p. 155-169, 1999.

SOCIEDADE BRASILEIRA DE SILVICULTURA - SBS. Estatísticas. [17/10/2000]. (http://www.ipef.br/sbs/ estatisticas)
SWITZER, G. L.; NELSON, L. E. Nutrient accumulation and cycling in loblolly pine (Pinus taeda L.) plantations ecosystems: the first twenty years. Soil Science Society of America Proceedings, v. 36, p. 143-147, 1972.

SARRUGE, J. R.; HAAG, H. P. Análises químicas em plantas. Piracicaba: Escola Superior de Agricultura "Luiz de Queiroz", 1974. 56 p.

TEIXEIRA, J. L. et al. Biomassa e conteúdo de nutrientes de duas espécies de eucalipto em diferentes ambientes do Médio Rio Doce, MG. Revista Árvore, v. 13, n. 1, p. 34$50,1989$.

RAIJ, B. van; QUAGGIO, J. A. Métodos de análise de solos para fins de fertilidade. Campinas: Instituto Agronômico, 1983. 32 p. 